\title{
Large Cell Acanthoma of the Conjunctiva: Clinicopathologic and Immunohistochemical Features
}

\author{
Frederick A. Jakobiec ${ }^{a}$ Paula Cortes Barrantes ${ }^{a} \quad$ Lina Ma $^{a} \quad$ John Mandeville ${ }^{b}$ \\ ${ }^{a}$ David G. Cogan Laboratory of Ophthalmic Pathology, Massachusetts Eye and Ear Infirmary/Harvard Medical

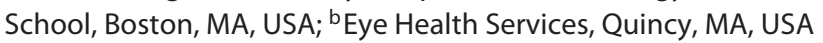

\section{Established Facts}

- Cutaneous large cell acanthoma (LCA) occurs uncommonly and mainly in sun-exposed skin.

- The lesion is composed of large keratinocytes with benign cellular features.

- LCA in a mucous membrane is extremely rare.

\section{Novel Insights}

- LCA can develop in the conjunctiva and frequently recurs.

- Histopathologically, LCA differs from conjunctival squamous dysplasias due to the absence of overwhelming atypia.

- Immunohistochemical evaluation discloses negative CK-7, positive CK-14 (basal cells), positive CK17, and low Ki-67/p53 restricted to basal and parabasal cells.

- Immunohistochemistry can help to separate LCA from dysplasias on an objective basis.

\section{Keywords}

Large cell acanthoma · Conjunctiva · Skin · Mucous membranes $\cdot$ Cytokeratins $\cdot$ p53 $\cdot$ Ki-67

\section{Abstract \\ Large cell acanthoma (LCA) was first described as a lesion on sun-exposed skin. All LCAs feature keratinocytes twice the size of normal cells (cytomegaly). Although infrequently di- agnosed in the skin, it has been even more rarely described by ophthalmic pathologists in the eyelid skin and the con- junctiva. This report describes the third case of a conjunctival}

epithelial LCA, with the first published clinical photograph highlighting its leukoplakic and well-circumscribed character, as well as the most thorough analysis of the immunohistochemical features of this lesion. It is contrasted with squamous dysplasias and papillomas of the conjunctiva. A review of previous conjunctival LCA lesions discloses frequent recurrences after initial surgery and the remote but real potential for squamous dysplastic transformation. Immunohistochemical stains for certain cytokeratins, p53, and Ki-67 (proliferation index) will in the future be particularly helpful in establishing an early and accurate diagnosis of conjunctival LCA.

\section{KARGER}

E-Mail karger@karger.com

www.karger.com/oop
(C) 2019 S. Karger AG, Basel

Frederick A. Jakobiec, MD, DSc
David G. Cogan Laboratory of Ophthalmic Pathology

Massachusetts Eye and Ear Infirmary

243 Charles Street, Suite 328, Boston, MA 02114 (USA)

E-Mail Fred_Jakobiec@ MEEI.Harvard.edu 


\section{Introduction}

Large cell acanthoma (LCA) is an uncommon cutaneous epidermal lesion [1-5]. It arises extremely infrequently in the conjunctiva $[6,7]$ and has never been reported in the oral mucosa or other mucous membranes. The purposes of this study are (1) to provide the first prebiopsy photograph of a conjunctival LCA, (2) to describe its histopathologic features, and (3) to report the investigative results of an expanded immunohistochemical panel of biomarkers. A handful of previous publications dealing with eyelid and conjunctival LCAs will also be carefully reviewed.

\section{Case Report}

\section{Clinical History}

A 70-year-old Asian woman presented with the chief complaint of a "white spot" growing on the inside of her lower eyelid over the course of 2 weeks, and it was allegedly rapidly enlarging. Her symptoms included itching, foreign body sensation, and tearing. Her medical history included hypertension and anxiety, without a history of cancer. Her ophthalmic history was significant for blepharitis with chalazia, eyeliner tattooing, and dry eyes. On examination, vision was 20/30 in each eye. There was no eyelid edema, erythema, conjunctival injection, or chemosis. There was a dense punctate keratopathy of the inferior corneas, bilaterally, consistent with dry eyes. An elevated, white, hyperkeratotic plaque was noted in the inferior palpebral conjunctiva region of the medial left lower eyelid that extended onto the inferior bulbar conjunctiva. The lesion measured roughly $8 \times 10 \mathrm{~mm}$ and was fixated to the tarsus. A biopsy was performed. Initially, it was interpreted microscopically as "hypertrophic actinic keratosis," focally bordering on squamous cell carcinoma in situ. A wide surgical excision was performed with reconstruction of the inferior fornix. The histopathologic diagnosis was a "squamous cell carcinoma, well differentiated, superficially invasive." The patient was referred for interferon therapy. The biopsy slides were sent to the Massachusetts Eye and Ear Infirmary for consultation.

\section{Histopathologic and Immunohistochemical Findings}

According to the outside pathology report, the specimen was received in buffered formalin in the laboratory. It was a single piece of tan-white soft tissue measuring $1.1 \times 0.6 \times 0.2 \mathrm{~cm}$. The specimen had been bisected longitudinally and submitted for histopathologic evaluation.

Re-evaluation of the microscopic slides at the Infirmary disclosed at medium power a thickened zone of the conjunctival epithelium that was composed of large, deeply eosinophilic cells in its lower half, above the basement membrane. Paler and swollen cells were seen in the upper half of the lesion (Fig. 1b). Higher-power microscopy revealed several layers of bland basaloid cells and large suprabasal keratinocytes twice the size of normal squamous cells, with capacious eosinophilic cytoplasm and centrally placed round nuclei. The latter possessed prominent hematoxyphilic nuclei without atypia (lacking hyperchromasia or bizarre configurations)
(Fig. 1c). At the surface, the cells displayed incipient parakeratosis with pyknotic nuclei and more pronounced cytoplasmic eosinophilia. More advanced degeneration of the surface cells suggestive of ghost cells (superficially mimicking those of a calcifying pilomatrixoma) was also observed in some regions of the lesion (Fig. 1e). The periodic acid-Schiff (PAS) stain disclosed the presence of abundant, positively staining granules in the suprabasal keratinocytes (analogous to the Malpighian layer of the cutaneous epidermis) that were sensitive to diastase digestion, indicative of glycogen. The upper half of the thickened epithelium did not contain such granules (Fig. 1f). An abrupt interface was discovered at the edges of the lesion abutting the uninvolved conjunctival epithelium. (Fig. 2a) Minimal numbers of chronic inflammatory cells were located beneath the lesion in the substantia propria.

The immunohistochemical staining (Table 1) displayed negativity to cytokeratin (CK)-7 (Fig. 2b, left panel) (in contrast to positivity in normal conjunctival epithelium) and scattered CK-10 positivity in the upper half of the lesion (Fig. 2b, right panel) (in contrast to the CK-10 total negativity in normal conjunctival epithelium). CK-14 immunostained the full thickness of the lesion with notable strong positivity in the superficial cells (Fig. 2c) (in contrast to CK-14 limited to staining only the basal cells in normal epithelium). CK-17 stained the full thickness of the lesion (Fig. 2d) (in contrast to complete negativity in normal conjunctiva). p53 immunostained the basal and parabasal cells but not the upper two thirds of the lesion, as encountered in dysplasias. p16 failed to reveal any positivity in the epithelium. Finally, Ki-67 nuclear positivity, signifying premitotic synthesis of DNA, was confined to the basal region of the lesion and was not observed at higher levels of the epithelium as is typical of dysplasias and carcinomas in situ.

\section{Discussion}

LCA of the skin is described clinically as an asymptomatic, well demarcated, somewhat elevated hyperkeratotic cutaneous plaque, usually around $10 \mathrm{~mm}$ in diameter, with a slow evolution over months to years [1-6]. It appears most often in middle-aged and elderly females, typically in sun-exposed skin (the face, upper extremities, trunk, and to a lesser extent, in the lower extremities). Most patients have single lesions, but there have been reports of multiple or eruptive LCAs $[8,9]$.

Histopathologically, LCA is sharply circumscribed when abutting the adjacent normal epithelium. The epidermis shows acanthosis and surface orthohyperkeratosis or parakeratosis with a prominent granular layer [2]. Alternatively, the surface cells may infrequently show degenerative changes approximating clear or ghost cells with pyknotic nuclei. The predominating constituent cells arelarge, polygonal epidermoid acanthocytes around twice the size of the normal surrounding keratinocytes. The capacious eosinophilic cytoplasm contains vesicular nuclei with prominent nucleoli. The intercellular spaces can be widened, revealing stretched intercellular attach- 
Fig. 1. Clinicopathologic features of conjunctival large cell acanthoma. a A 70-yearold woman developed a sharply circumscribed, white, "leukoplakic" lesion in the left lower palpebral and forniceal conjunctiva that had caused a foreign body sensation with tearing. b Medium-power photomicrograph of the central portion of the lesion reveals substantial thickening of the epithelium. The lower half is eosinophilic, while the upper half appears pale and swollen. Note the absence of a papillary architecture (periodic acid-Schiff [PAS], $\times 2$ ). c The basal region is composed of large eosinophilic cells (cytomegaly) with centrally placed, round, vesicular nuclei possessing prominent nucleoli. No atypical cells with nuclear hyperchromasia are seen (hematoxylin and eosin $[\mathrm{H} \& \mathrm{E}], \times 40)$. d The superficial and surface cells display more intense cytoplasmic eosinophilia indicating incipient nuclear pyknosis and parakeratosis $(\mathrm{H} \& \mathrm{E}, \times 40)$. e More advanced nuclear pyknosis with delamination of dyscohesive, marginally vital surface cells $(\mathrm{H} \& \mathrm{E}$, $\times 20)$. $\mathbf{f}$ The PAS stain discloses a zone of PAS-positive glycogen-rich cells (between arrows) and an absence of glycogen in the pale surface cells. The glycogen disappeared with diastase predigestion (PAS, $\times 10)$.
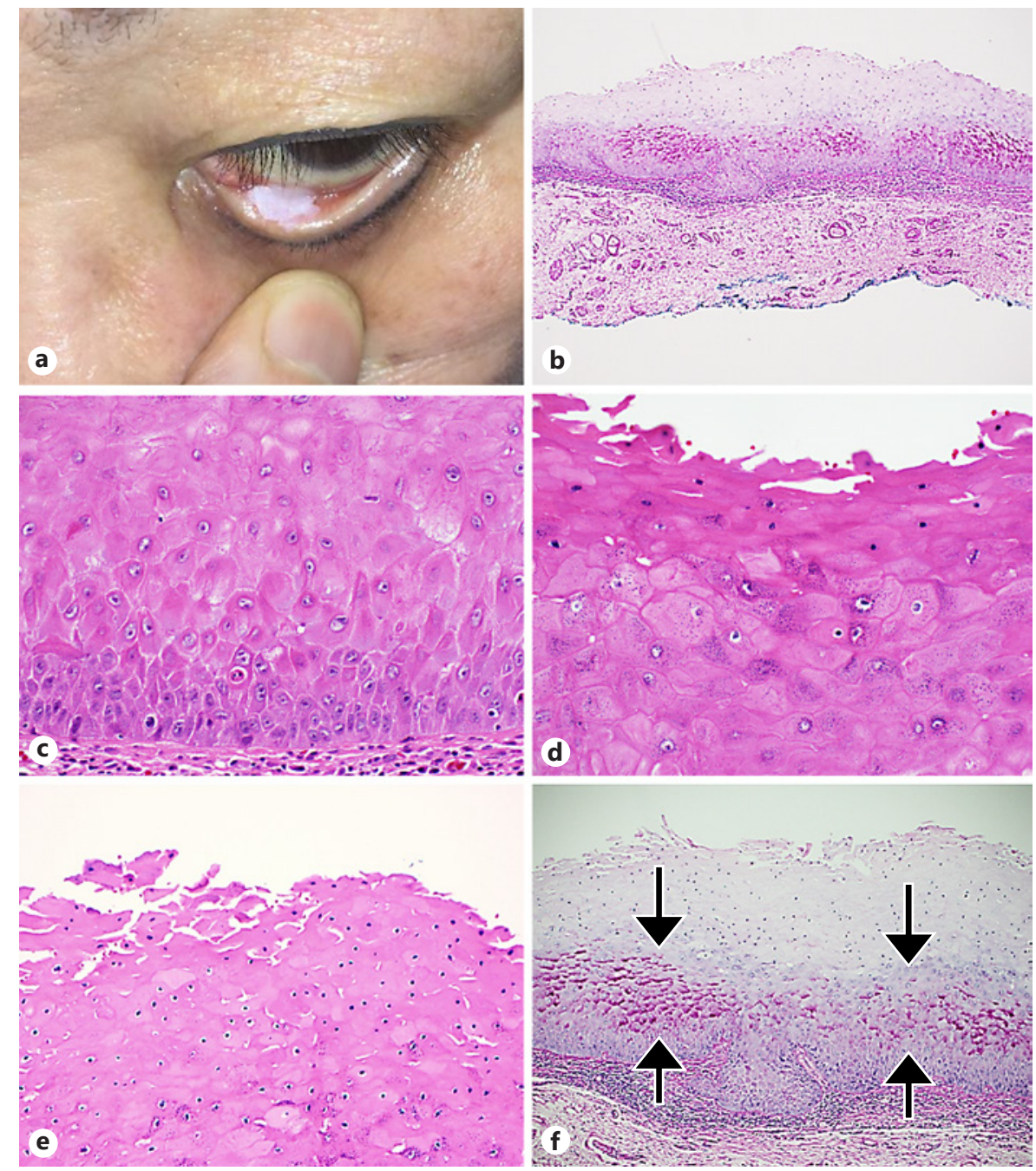

ments (desmosomes). Either low papillae or no papillomatosis may be appreciated.

The PAS stain reveals an intact basement membrane and diastase-sensitive cytoplasmic granules (glycogen) in the epidermal Malpighian layer. Mitotic activity is virtually absent. Subepithelial inflammation is scant and is not a diagnostic criterion. Basal cells (germinative cells resting on the basement membrane) may display hyperpigmentation allegedly without an increase in the number of melanocytes [10]. However, a more recent study utilizing immunohistochemistry has established that some lesions exhibit an increased number of melanocytes demonstrated with the HMB45 stain [1]. Hypochromic and achromic LCA variants have also been clinically and microscopically described [11]. Most dermatopathologic studies have confirmed the fundamentally non-dysplastic nature $[12,13]$ of cutaneous LCA with no example of a malignant transformation having been described to date. Treatment has been complete surgical excision with a modest removal of normal surrounding skin to create tumor-free margins.

From the ophthalmic perspective, only five lesions of LCA have been documented: two in the eyelids contained in one report published in 1991 [14] and three (including the present one) in the conjunctiva $[6,7]$. The histopathologic features of these lesions fulfilled the diagnostic microscopic criteria described above for cutaneous LCAs. The two cases of eyelid LCAs developed in men aged 62 and 71 years. Both involved the right upper eyelid and developed either over 3 months or 2 -year periods. They had caused ocular irritation and a foreign body sensation. The eyelid lesions were clinically described as whitish, placoid, pale, sharply outlined, and manifesting variable degrees of papillomatosis. One lesion recurred a year after 
Fig. 2. Histochemical and immunohistochemical features of large cell acanthoma (LCA) cells. a The periodic acid-Schiff (PAS) stain clearly demonstrates an abrupt interface between the LCA cells on the left and normal conjunctival epithelial cells on the right. The LCA cells are at least twice the size of the normal keratinocytes, mostly due to their abundant cytoplasm. Note that the LCA cells have a low nuclear-tocytoplasmic ratio. The arrows indicate a non-violated basement membrane. Chronic inflammation is present in the subepithelial substantia propria (PAS, $\times 40)$. b Left panel: CK-7 immunohistochemical staining, normally positive in conjunctival epithelium, is negative in the LCA cells. Right panel: CK-10 discloses scattered positivity in the LCA cells. There is negativity in normal epithelium. Chronic inflammation is present in the subepithelial substantia propria $(\times 10)$. c CK-14 immunostains the full thickness of the LCA cells with more intense staining of the surface parakeratotic cells. Normal conjunctiva displays CK-14 staining that is restricted to the basal and parabasal keratinocytes $(\times 10)$. d CK-17 is full thickness positive in the LCA cells, whereas it is totally negative in normal epithelium. The arrows indicate unstained basal cells $(\times 20)$. e p53 nuclear immunostaining is limited to the basal and parabasal cells, in contrast to the higher-level staining observed in dysplasias. The extent of the upward staining depends on the severity of the dysplastic process $(\times 10)$. f Nuclear Ki67 staining is tightly restricted to the base epithelial cells as in normal epithelium. In dysplasias, the Ki-67 positivity is found at higher levels according to the severity of the lesion $(\times 10)$.
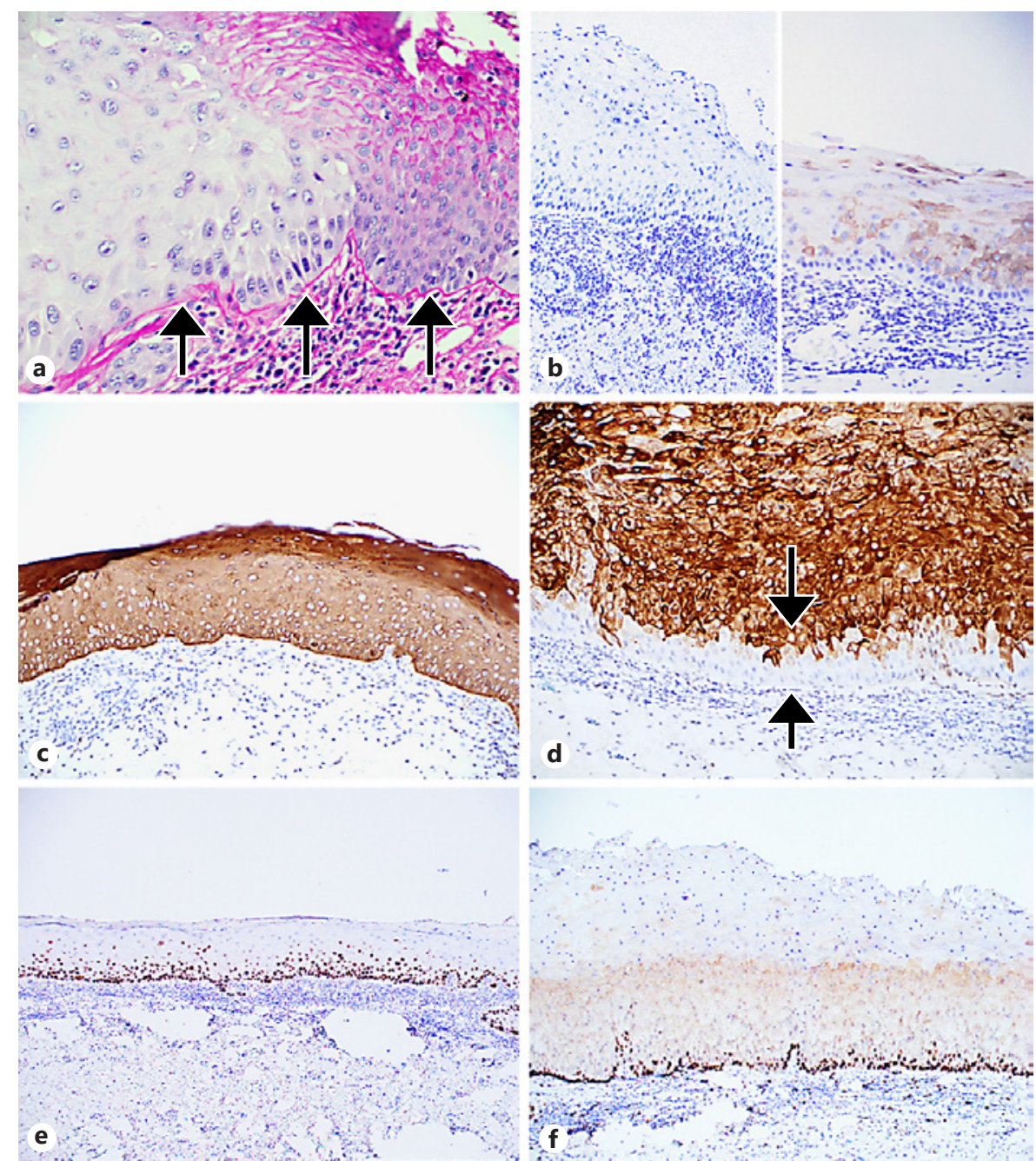

a subtotal initial excision. No atypical cytologic features other than cytomegaly were detected in the recurrence.

The two lesions microscopically displayed hyperkeratosis and hypergranulosis; also evident were occasional atypical cells with larger vesicular nuclei possessing enlarged nucleoli. A low nuclear-to-cytoplasmic ratio was maintained and there were no mitotic figures in the lesion. Scattered dyskeratotic cells were randomly distributed among the acanthoma cells. Also seen were extensions of acanthoma cells into the infundibular or outer sheath portions of the hair follicles and club-like expansions of the rete ridges that protruded into the upper dermis without breaching the epidermal basement mem- brane. An addendum at the end of the report briefly described a lesion that the authors had recently seen after their paper had been accepted for publication [14]. It developed in a 56-year-old man who had a left medial canthal lesion with the classic microscopic features of an LCA. No clinical follow-up in this case was available.

In addition to the eyelid examples, there are two previously reported LCAs of the conjunctiva. The first appeared in the dermatopathology literature in 2010 [6]. A 49-year-old man had irritation and a foreign body sensation in the right eye caused by a $3.7 \times 3.7 \mathrm{~mm}$, slightly raised, temporal, juxtalimbal, and epibulbar lesion with dilated vessels that spared the cornea. From the convinc- 
ing microscopic description, one can also infer, in the absence of a preoperative photograph, that the lesion was clinically well demarcated and probably whitish (leukoplakic) due to the microscopic evidence of surface hyperorthokeratosis and hypergranulosis. This lesion was remarkable because it recurred within 8 months, despite the microscopic assessment that the original surgical margins were negative. There was no evidence of subepithelial invasion at this time. After excision of the first recurrence, the lesion reappeared a second time. On this occasion, it extended $1.5 \mathrm{~mm}$ onto the cornea with histopathologic features that were incontrovertibly those of a carcinoma in situ. Remnants of the earlier LCA in the form of persistent benign cells were detected in the specimen. There was no hyperorthokeratosis or hypergranulosis produced by the tumor cells. The surgical margins were deemed to be tumor free, but no further follow-up information was provided after the second re-excision.

The second published conjunctival lesion appeared in 2013 [7] and concerned a 51-year-old man who developed over the prior 12 months a left nasal, opalescent, epibulbar juxtalimbal lesion that had created some blurring of vision and a foreign body sensation. The lesion was debrided but a microscopic examination was not requested. Six months later, the lesion recurred and involved more of the corneal surface. After 6 years, the lesion recurred a third time. A final nasal limbal recurrence developed 6 years later. During 6 months of follow-up after the final recurrence, the lesion had not reappeared. All of the recurrences in this case displayed the same benign histopathologic features of a LCA.

The conjunctival case reported herein is the third case and the first with a preoperative clinical photograph. Ophthalmic examination revealed a white, hyperkeratotic lesion in the lower palpebral/forniceal conjunctiva that was sharply demarcated from the surrounding normal conjunctiva. The histopathologic features of this LCA are fully illustrated in the Results section of this article and correspond to standard diagnostic criteria.

The immunohistochemical staining results in the present case provide valuable adjunctive data that allow the diagnosis in questionable lesions and by facilitating separation of the entity from conjunctival squamous dysplasias and carcinomas in situ (Table 1). First, CK-7, normally expressed in the conjunctiva, was negative in the present case and in the earlier one published in 2013 [7]. CK-10 was focally positive, whereas it is totally negative in normal conjunctiva. CK-14 stained the full thickness of the lesion in contrast to the usual result of basal and parabasal staining. CK-17 is negative in the normal con-

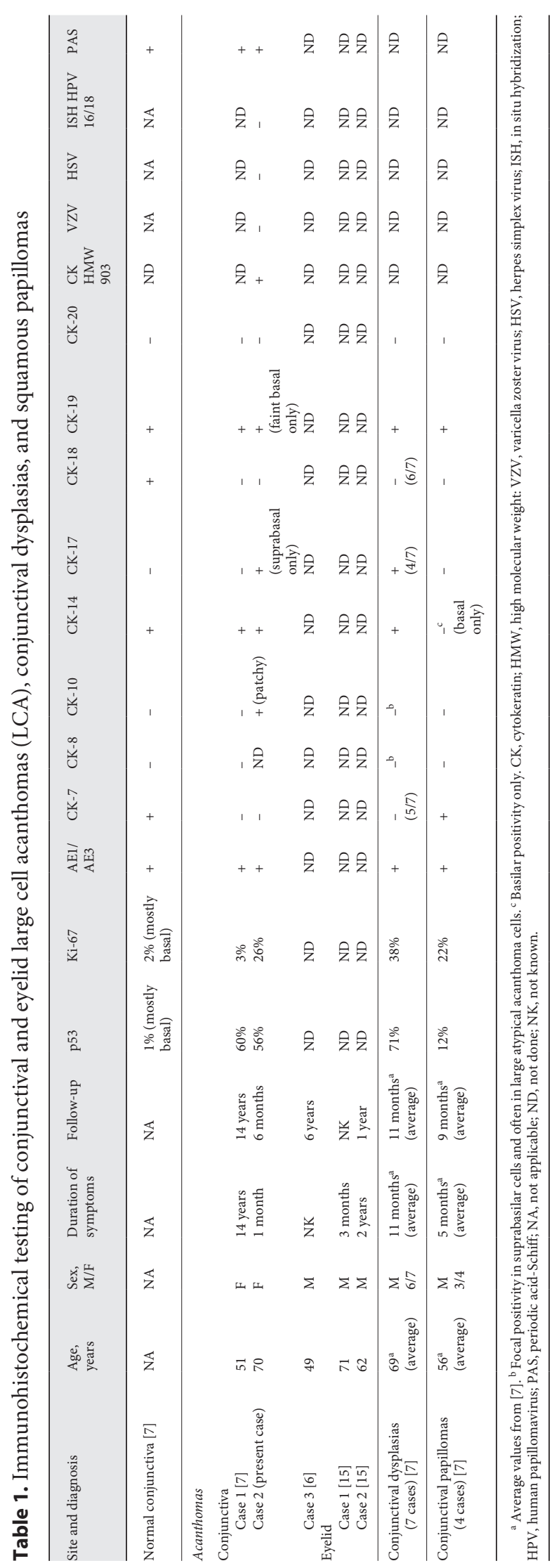

Jakobiec/Cortes Barrantes/Ma/Mandeville 
junctiva but was full thickness positive in the present case, in common with dysplasias and carcinomas in situ. It was negative, however, in a previously reported case, which recurred multiple times. It is likely that during the recurrences, further genetic shifts occurred that resulted in loss of expression of this biomarker, as the last recurrence was the one that was studied immunohistochemically [7].

Heavy-weight CK-903 was positive in the present lesion but negative in the normal conjunctiva adjacent to the lesion. This marker was not included in the previous conjunctival LCA immunohistochemical study [7].

In support of the benignity of the present and an earlier conjunctival case is the finding that Ki-67 nuclear labelling was found only in the basal and occasional parabasal cells, whereas it reaches higher levels in dysplasias and carcinomas in situ, correlating with the severity of the process. Further reinforcing the benign nature of these lesions both before and after multiple recurrences is the clear-cut restriction of p53 nuclear staining to the basal and parabasal cells, in contrast to true squamous premalignant lesions, in which staining is found at variable higher levels of the lesion. p53 is a suppressor gene which, when it mutates, results in the accumulation of an abnormal protein detectable by immunohistochemistry.

Regarding pathogenesis, very little solid scientific and investigative data are available. Immunohistochemical studies provide no reliable insights. Papillomavirus 6 has been detected in one cutaneous case $[6,9,15]$, but in our case, antisera against herpes simplex, herpes zoster, and human papillomavirus 16/18 antigens failed to reveal any positivity [16]. Most authorities are of the opinion that p16 is a highly sensitive screening test for any infection by human papillomavirus types, and in the current case, it was negative $[17,18]$. Polyploidy has been established to occur in LCA $[19,20]$ and may explain the phenomenon of clonal cytomegaly. A role for sun-exposure in conjunctival LCA lesions is a possibility, but is only a hypothesis at this moment [10]. The current patient's lesion was found in non-sun-exposed tarsal conjunctiva; this feature would obviously rule out ultraviolet radiation as a cause of the lesion.

In terms of differential diagnosis, in the past, cutaneous LCA was often considered to be microscopically a form of epidermal dysplasia, actinic keratosis, Bowen's disease, or solar lentigo $[2,3,10,21]$. A recent study analyzes the possibility that LCA is a variant of solar lentigo vis-à-vis seborrheic keratosis and actinic keratosis [21]. There were no apparent differences noted between LCA and solar lentigo in an analysis employing CK-10, CK-16, Bcl-2, and Ki-67 expression. The conclusion was that

Conjunctival Large Cell Acanthoma
LCA is a hypertrophic immunophenotypic variant of solar lentigo with morphologic differences related to cell kinetics.

In our opinion, most ophthalmic pathologists have been unaware of the lesional category of LCA and hence have probably underdiagnosed and/or misassigned it by placing it in the spectrum of squamous dysplasia and carcinomas in situ. The immunohistochemical findings of limited basal region positivity of p53 and Ki-67 forcefully militates against this proposition [7]. The white sponge nevus is an autosomal dominantly inherited mucosal leukoplakic-appearing lesion predominantly in the oral mucosa but also less commonly in other extraoral mucosae $[2,22-24]$. It could be considered in the differential diagnosis of conjunctival LCA, but in fact has never been reported in this location. The white sponge nevus is clinically stable, often can appear early in life and does not progress to a premalignant or cancerous lesion.

Microscopically, it is a papillary lesion composed of a thickened epithelium with a surface keratin layer (unassociated with a granular cell layer) and large, swollen, vacuolated keratinocytes normally seen in the oral mucosa but not encountered in the conjunctiva. Its malignant potential has not been documented. On the other hand, LCA has never been reported in a mucosa other than the conjunctiva. Lastly, pseudoepitheliomatous hyperplasias of the conjunctiva evince hyperkeratosis and parakeratosis that develop rapidly, as opposed to the slow evolution of LCA.

The management of periocular LCA, and specifically lesions of the conjunctiva, should be wide local excision. One of two eyelid lesions and two of the three conjunctival lesions recurred after initial surgery. The third reported conjunctival case had insufficient follow-up (only 6 months) to conclude that it was completely excised. It should be remembered that two previous conjunctival lesions recurred multiple times $[6,7]$. One late recurrence displayed unequivocal microscopic evidence of a carcinoma in situ [6]. This development has not been reported anywhere in the skin nor in the two other conjunctival lesions (including the present case) up to date. Therefore, careful dissection with wide margins must be performed for conjunctival LCA, like that done for dysplasias and carcinomas in situ; if necessary, adjunctive cryotherapy may be added [25]. Topical chemotherapy to prevent recurrences of incompletely excised conjunctival lesions would probably be ineffectual in view of the low mitotic index exhibited by the lesions where there are no histopathologic signs of a true premalignant transformation. 


\section{Statement of Ethics}

The authors have no ethical conflicts to disclose.

\section{Disclosure Statement}

The authors have no conflicts of interest to declare.

\section{Funding Sources}

Department of Ophthalmology Research Fund, Massachusetts Eye and Ear Infirmary.

\section{Author Contributions}

Frederick A. Jakobiec: main author, responsible for supervision and conceptual guidance. Paula Cortes Barrantes: responsible for data summary, preparation, and illustrations. Lina Ma: responsible for library research and illustrations. John Mandeville: provided the case report and clinical photographs.

\section{References}

1 Mehregan DR, Hamzavi F, Brown K. Large cell acanthoma. Int J Dermatol. 2003 Jan; 42(1):36-9.

2 Elder DE, Elenitsas R, Rosenbach M, Murphy GF, Rubin AI, Xu X. Lever's Histopathology of the Skin. 11th ed. Philadelphia, USA: Wolkers Kluwer; 2015.

3 Sánchez Yus E, de Diego V, Urrutia S. Large cell acanthoma. A cytologic variant of Bowen's disease? Am J Dermatopathol. 1988 Jun; 10(3):197-208.

4 Sánchez Yus E, del Rio E, Requena L. Largecell acanthoma is a distinctive condition. Am J Dermatopathol. 1992 Apr;14(2):140-7.

5 Shahriari N, Grant-Kels JM, Rabinovitz HS, Oliviero M, Scope A. In vivo reflectance confocal microscopy features of a large cell acanthoma: report of a case. Dermatol Pract Concept. 2016 Jul;6(3):67-70.

6 Ghazi NG, Patel BS, Olsakovsky LA, White K, Patterson JW, Carter B. A conjunctival lesion with histological features similar to large-cell acanthoma of the skin. J Cutan Pathol. 2010 Oct;37(10):1103-6.

7 Jakobiec FA, Mendoza PR, Colby KA. Clinicopathologic and immunohistochemical studies of conjunctival large cell acanthoma, epidermoid dysplasia, and squamous papilloma. Am J Ophthalmol. 2013 Oct;156(4): 830-46.

8 Rabinowitz AD. Multiple large cell acanthomas. J Am Acad Dermatol. 1983 Jun;8(6): $840-5$.
9 Berger T, Stockfleth E, Meyer T, Kiesewetter F, Funk JO. Multiple disseminated large-cell acanthomas of the skin associated with human papillomavirus type 6. J Am Acad Dermatol. 2005 Aug;53(2):335-7.

10 Rahbari H, Pinkus H. Large cell acanthoma. One of the actinic keratoses. Arch Dermatol. 1978 Jan;114(1):49-52.

11 Ambrojo P, Aguilar A, Requena L, Sánchez Yus E. Achromic verrucous large cell acanthoma. J Cutan Pathol. 1990 Jun;17(3):182-4.

12 Rabinowitz AD, Inghirami G. Large-cell acanthoma. A distinctive keratosis. Am J Dermatopathol. 1992 Apr;14(2):136-8.

13 Weinstock MA. Large-cell acanthoma. Am J Dermatopathol. 1992 Apr;14(2):133-4.

14 Chévez P, Patrinely JR, Font RL. Large-cell acanthoma of the eyelid. Report of two cases. Arch Ophthalmol. 1991 Oct;109(10):1433-4.

15 Lora V, Kanitakis J. HPV detection in 'largecell acanthomas': a word of caution. J Eur Acad Dermatol Venereol. 2009 Dec;23(12): 1468-9.

16 Odrich MG, Jakobiec FA, Lancaster WD, Kenyon KR, Kelly LD, Kornmehl EW, et al. A spectrum of bilateral squamous conjunctival tumors associated with human papillomavirus type 16. Ophthalmology. 1991 May;98(5): 628-35.

17 Afrogheh AH, Jakobiec FA, Hammon R, Grossniklaus HE, Rocco J, Lindeman NI, et al. Evaluation for High-risk HPV in Squamous Cell Carcinomas and Precursor Lesions Arising in the Conjunctiva and Lacrimal Sac. Am J Surg Pathol. 2016 Apr;40(4):519-28.
18 Buckley L, Jackett L, Clark J, Gupta R. HPVrelated Oropharyngeal Carcinoma: A Review of Clinical and Pathologic Features With Emphasis on Updates in Clinical and Pathologic Staging. Adv Anat Pathol. 2018 May;25(3): $180-8$.

19 Fand SB, Pnlus H. Polyploidy in benign epidermal neoplasia. J Cell Biol. 1970;476:59a.

20 Argenyi ZB, Huston BM, Argenyi EE, Maillet MW, Hurt MA. Large-cell acanthoma of the skin. A study by image analysis cytometry and immunohistochemistry. Am J Dermatopathol. 1994 Apr;16(2):140-4.

21 Fraga GR, Amin SM. Large cell acanthoma: a variant of solar lentigo with cellular hypertrophy. J Cutan Pathol. 2014 Sep;41(9):733-9.

22 Cai W, Jiang B, Yu F, Yang J, Chen Z, Liu J, et al. Current approaches to the diagnosis and treatment of white sponge nevus. Expert Rev Mol Med. 2015 May;17:e9.

23 García-Malinis AJ, Agón-Banzo PJ, Marigil MA, Gilaberte Y. Vulvar white sponge naevus in a girl. J Eur Acad Dermatol Venereol. 2016 May;30(5):855-6.

24 Sobhan M, Alirezaei P, Farshchian M, Eshghi G, Ghasemi Basir HR, Khezrian L. White sponge nevus: report of a case and review of literature. Acta Med Iran. 2017 Aug;55(8): 533-5.

25 Jakobiec FA, Rini FJ, Fraunfelder FT, Brownstein S. Cryotherapy for conjunctival primary acquired melanosis and malignant melanoma. Experience with 62 cases. Ophthalmology. 1988 Aug;95(8):1058-70. 